\title{
Enabling a Freely Accessible Open Source Remotely Controlled Robotic Articulator with a Neuro-Inspired Control Algorithm
}

\author{
https://doi.org/10.3991/ijoe.v13i01.6288
}

\author{
A. Vijayan, C. Nutakki, D. Kumar, K.Achuthan, B. Nair and S. Diwakar \\ Amrita University, Kerala, India \\ shyameamrita.edu
}

\begin{abstract}
Internet-enabled technologies for robotics education are gaining importance as online platforms facilitating and promoting skill training. Understanding the use and design of robotics is now introduced at university undergraduate levels, but in developing economies establishing usable hardware and software platforms face several challenges like cost, equipment etc. Remote labs help providing alternatives to some of the challenges. We developed an online laboratory for bioinspired robotics using a low-cost 6 degree-of-freedom robotic articulator with a neuro-inspired controller. Cerebellum-inspired neural network algorithm approximates forward and inverse kinematics for movement coordination. With over 210000 registered users, the remote lab has been perceived as an interactive online learning tool and a practice platform. Direct feedback from 60 students and 100 university teachers indicated that the remote laboratory motivated self-organized learning and was useful as teaching material to aid robotics skill education.
\end{abstract}

Index Terms-Remote labs, Robotic articulator, Neural Network, Open Source, ICT.

\section{Introduction}

Technological advances have led to an increase in the use of tools that augment hands-on learning via virtual and remote labs, providing affordable laboratory education to help students learn and practice experiments [1]. In this paper, we have developed a remote laboratory platform for robotics allowing students and teachers to use the platform for augmenting laboratory skill training. Remote laboratory is an Information and Communications Technology (ICT) approach that allow users to perform experiments using highly sophisticated and expensive instruments without having to be physically present in the laboratory [2]. Augmenting ICT-based skill training has been reported via animations, simulations, remote-triggered experiments and haptic technologies [3]. By sharing expensive laboratory equipment within a large number of users, remote labs provide affordable experimental experience to students from geographically challenged places [4]. Students using remote labs have been observed to repeatedly practice experiments attributing the advantages of remote labs on classroom constraints 
like reduced damage, resource or time unavailability [5]. A study based on feedback indicated that ease of learning was perceivably increased with usage of remote labs when compared to traditional learning methods like books and digital simulators [6]. Remote lab experiments have been used as practice material (activities and assignments) in addition to classroom assignments helping students develop improved problem solving skills[7]. ICT-enabled remote lab have been known to also reduce teacher work-load [5] and studies implicate their role [8] in helping students with selforganized learning through these technologies. In this paper, via field trials, we also explored the role of a remote lab as a novel platform that augmented skill education in students towards the understanding of design and function of low-cost robotic articulators with neuro-inspired control.

In recent years, significant advances have been observed in robotics, artificial intelligence and in the development of robotic control environments [9] which have also led to educational robotics as a component of STEM [10] subjects in schools and colleges. Robotics education implicates added economic cost and significant infrastructure requirements. Development of neuroprosthesis has gained attention as an emerging area of research that is being driven by innovation in healthcare and also by robotics education. Bioinspired design and keeping lower financial cost are challenges for novel neuroprosthestic designs. Research groups in India and developing nations have been trying to develop low-cost prosthetic devices and sustainable robots to improve human interface and to mimic human behaviour [11] [12]. Neural networks, a bioinspired approach inspired from the brain, have been employed as controllers that could learn and detect events [12]. Speech detection for interactive toys [13] currency counting [14] are some of the applications that uses neural networks on low cost devices. The third generation neural networks, spiking neural networks (SNN), are generalized mathematical models which are now used in the area of robotics to plan trajectory, predict co-ordinates, separate patterns, and classify tasks [15], [16]. SNN mimics the firing dynamics of the neurons giving more emphasis on timing and amount of the information processed. Bio-inspired control architectures with spiking neural networks (SNN) are now implemented on FPGA based boards to develop low cost, low power hardware for helping people with cervical spinal cord injury [17].

Conventional approaches of designing low-cost anthropomorphic robotic articulators with bio-inspired control mathematically reconstruct some of the complex kinematics of human arm without the neural coding geometries or spiking dynamics regulating spatiotemporal information. Studies have demonstrated prosthetic devices with inputs from rat brain circuits as bio-feedback controllers [18], [19]. Since 1970s, cerebellar supervised learning consisting of neural circuit models that optimally control via modulation of firing dynamics [20] have been proposed and have been used by implementing cerebellar network on VLSI chip[21]. Having a control algorithm employing neural firing dynamics instead of explicit kinematics could help biophysics and bioinspired robotics education.

This paper focuses on the design, implementation and pedagogical analysis of a low cost robotic arm that can be used an education platform for fundamental concepts in bio-inspired robotics. Low cost robotic articulator developed here lacked efficiency, accuracy and precision. These disadvantages allow studying the approximation roles of generalised spiking neural network algorithms that can represent and predict kinematics of the robotic articulator. The remote lab allowed students groups an access to laboratory practical skills in robotics. An assessment on the usability, usefulness and 
deploy-ability of this remote laboratory was also done on student and teacher groups. We have made the robotic articulator available freely via vlab.amrita.edu.

\section{Methodology}

\subsection{Architecture}

Remotely Controlled Robotic Articulator: A 6 degree-of-freedom (DOF) robotic articulator was designed and implemented to be used by students and teachers as a learning platform (Nair et al. 2008) in order to understand fundamental concepts in bio-inspired robotics. The articulator consisted of revolute joints that allowed roll and pitch movement with $20 \mathrm{~mm}$ precision and load capacity of $270 \mathrm{gms}$ and each joint could handle values from $0-180^{\circ}$ (Fig. 1A).

In the conventional method, the DH (Denavit-Hartenberg) technique [22] was employed to model the kinematics of the arm which used parameters $\alpha_{i}, a_{i}, d_{i}, \Theta_{i}$ to represents twist angle, link length, link offset and joint angle respectively for each joint of the robotic articulator [23]-[25] (Fig. 1C). We implemented forward kinematics [26] to calculate end-effector transformation using joint angle for the articulator (http://vlab.amrita.edu/?sub=3\&brch=257\&sim=1458\&cnt=3171) and inverse kinematics to find the joint positions when provided with the orientation and end effector coordinates (http://vlab.amrita.edu/?sub=3\&brch=257\&sim $=1459 \& \mathrm{cnt}=3170$ ). Each DOF was actuated via a servo motor (Fig. 1B) which was controlled by pulse width modulation signals of $20 \mathrm{~ms}$ time periods and a duty cycle of $1-2 \mathrm{~ms}$. A pulse of $1 \mathrm{~ms}$ was used to move the motor to 0 , a pulse of $1.5 \mathrm{~ms}$ was used to move the motor to 90 and a pulse of $2 \mathrm{~ms}$ was used to move the motor to 180 . Sequential pulse inputs maintained the position of the articulator. The articulator was controlled remotely over internet and included a live video feed.

A dataset of 110 data points was generated from the robotic articulator to train the spiking neural network algorithm. A data point contained 9 attributes (6 motor values and an end effector Cartesian coordinates). In the initial part of the study the dataset was used to train a spiking neural network (cross-validated with a percentage split method).
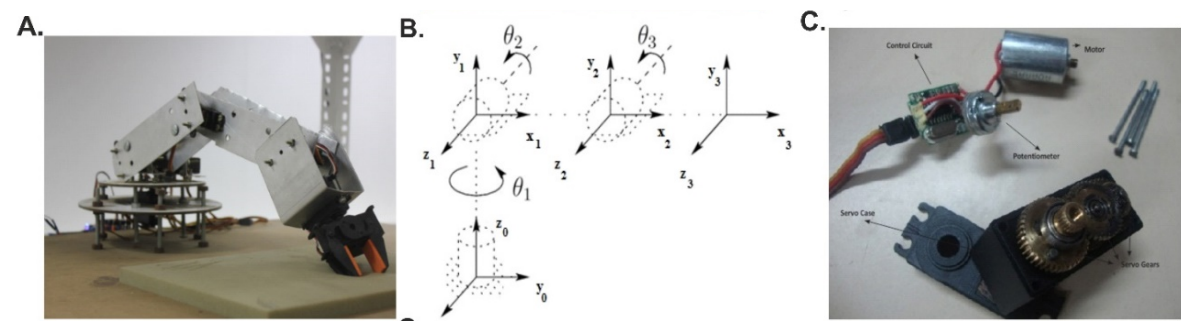

Fig. 1. Deploying a low-cost robotic arm as a remote lab: A. 6 DOF robotic manipulator used as an equipment for remote lab B. Axis representation for the joint angles. C. Servo motor components 

Neuro-Inspired Control Algorithm

Remote laboratory: Remote lab was developed on the virtual laboratory collaboration and accessibility platform (VLCAP) [27] platform that provided integration, support and secure access to remote learners. Learning related content such as theory, procedure, quiz, assignment and references were posted as tabs. Users may access all learning related tabs in the remote lab. User authentication was included to ensure single user access at any given time. Authenticated users could $\log$ on to the remote panel and scheduler. Slot-booking deployed in the system allowed users to reserve their required time slots so that the equipment was available for the user at that particular time. The authentication details were stored and used as a metric to calculate the usage time of the device as per user. An USB video device connected to the server provided live streaming of the triggered experiment.

We implemented the remote labs using two different approaches (Fig. 2). Preliminary implementation (Fig. 3) of remotely controlled labs was based on commercial software and hardware [28]. Programming was done using National Instruments LabVIEW, a commonly used control, simulation and automation software. Data acquisition cards were used to acquire data from an XML-based input by a user who altered or moved the virtual control knob or the slider on the GUI. Data transmission was regulated to reduce requirements of network bandwidth and the quantity of data transmitted assuming users from remote villages in India had lower bandwidth. We even reduced the video frames per second to 7 to keep experimental bandwidth low at the user end.

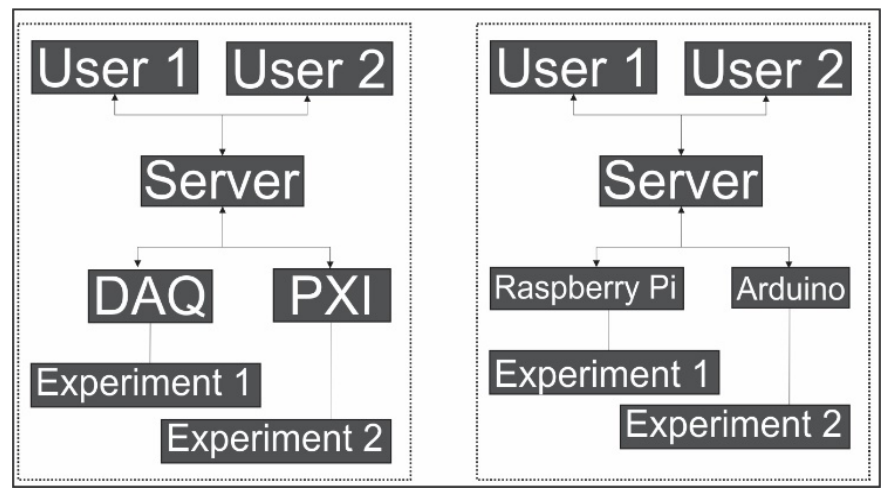

Fig. 2. Remote lab deployments with proprietary (left) and open-source implementations (right).

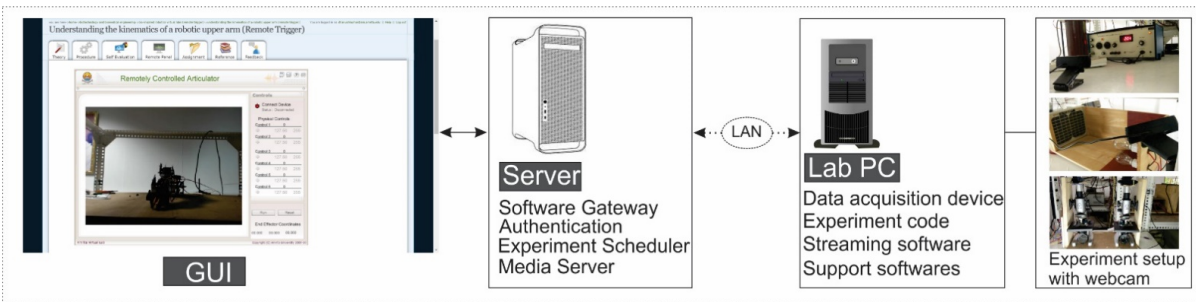

Fig. 3. Implementation and Architecture of Remote lab available via vlab.amrita.edu 
As an academic challenge, we followed the Free and Open Source Software (FOSS) implementation as a second approach, where we used an ARM processor based Raspberry Pi as controller and a JAVA-based web server for communication purposes. In this implementation, the experiment set-up was connected to the ARM processor which was linked to the lab server through a wireless network connection. Embedded ARM processor has protocols to retrieve and transmit data over the internet which makes the triggering of experiments easier. Controller for the robotic arm was modelled on the software as an artificial neural network based on the cerebellar architecture. When a user sends an input signal through GUI, the control program communicates a corresponding trigger to the remote equipment.

Remote lab GUI allows users to control knobs and buttons in GUI and thereby adjust the instrument setting [29]. When user accesses an experiment, http GET request was sent to the web server that fetches the corresponding file and sends it to the client browser. User can select an input and click the "Run" button, a XML coded instruction is sent to the lab server. Lab server decodes the message and transfers it to the data acquisition card and the control program then controls the experiment. All the received signals were processed by the bioinspired controller which was then send to remote device through the microcontroller. Remote equipment was triggered and the output raw data was collected from the data acquisition device. Control software processes the output data and sends back to the web server that transfers output to the user GUI over the internet. Results were recorded and transmitted to user GUI which can be exported (comma separated values or JPEG formats) for further use.

We perceived that the FOSS implementation allowed us to substitute the cost of proprietary software although configuring hardware and their complexity [3] needed to be handled independently.

\subsection{Bioinspired Controller}

Cerebellum has been known for sensorimotor control which is attained by its structure, function and modular nature. We modelled inputs as in sparse coded patterns of granular layer. As in a rat cerebellum, each granule cell is known to receive around 3-5 mossy fibres[30]. Sparse coded granule cell activities are integrated and the spike information is passed to the Purkinje cell. This allows to perceive the cerebellum granular layer and the Purkinje cell as a pattern separator and perceptron.

The bioinspired controller used to control the arm was based on a cerebellar architecture (Fig.4.) with spiking neurons represented by Adaptive Exponential Integrate and Fire (AdEx) [31] models. Kinematic parameters obtained from the robotic articulator were normalised and converted to spiking information using different encoding methods [32]. Spiking patterns obtained for each input was divided into $20 \mathrm{~ms}$ time bins and then rate coded by counting the number of spikes in each bin. Studies have shown that there was a maximum of 15 spikes in 50ms [33]. 18 granule neuron models were created for each time-bin. Input quantisation was kept as 4 so as to incorporate properties such as generalisation and learning interference.

If there were 2 spikes in $50 \mathrm{~ms}$ then $2^{\text {nd }}-5^{\text {th }}$ granule neurons in then model were excited to fire. The network circuit consisted of 3600 neuron models from among which around 100 neuron models were activated based on the pattern thus acting as pattern separator. The whole network was build based on a lookup table which adapts the 


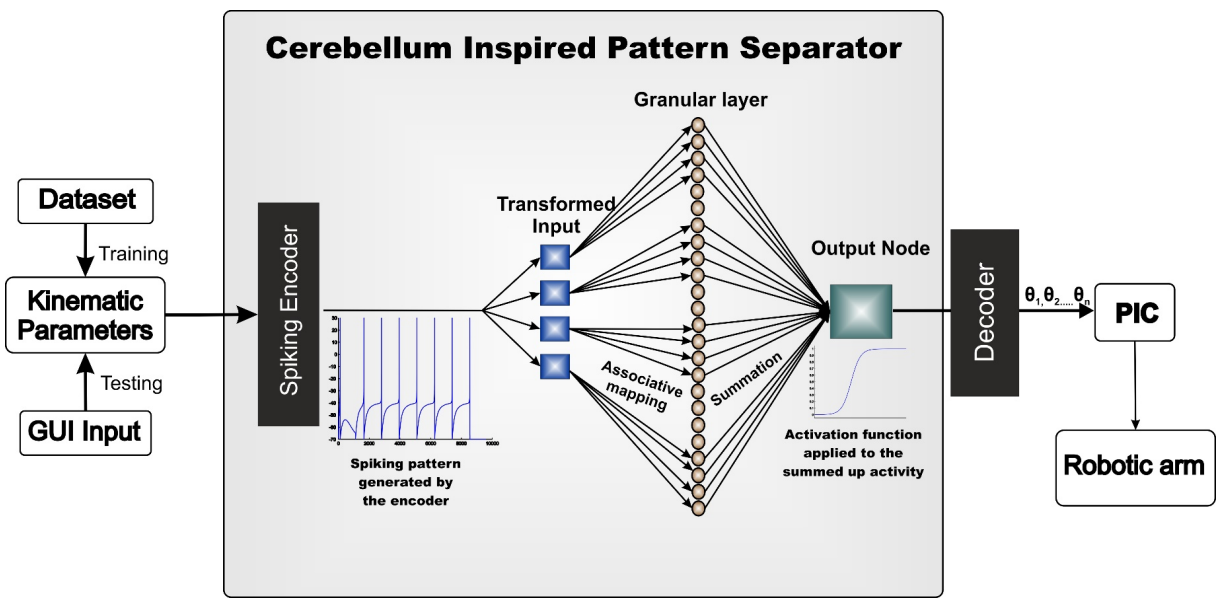

Fig. 4. Cerebellum Inspired Pattern Separator for GUI based Remote labs

Cerebellar Motor Articulation Control (CMAC) [15]. The activity of the granule neuron models was integrated and then a threshold was applied to classify the pattern, following which it was passed through the decoder. Decoder converted the spiking signals into the joint angles (manuscript in preparation) and given to the microcontroller. Learning was modelled using Hebbian mechanisms, adapting at the granular layer model to output node connections allowing the robotic manipulator to reach the user assigned target, while adjusting the motor angles by a feedback mechanism. During the training phase, the datapoints collected from the GUI as csv (comma separated value) file were used to set the connection weights of the network. Test the learnt classifier was performed directly by taking user-based input from the GUI and predicting the trajectory.

\subsection{Cost Analysis on Implementation of Open Source Model over Non Open Source Model}

In order to evaluate the efficacy of our deployment of online laboratories, economic factors were assessed. Sustained usability of such platforms rely mostly on the cost related to it [34]. Cost related attributes including experimental setup implementation, deliverability, and maintenance of lab resources were analysed. A survey on these key factors that governed implementation and deployment of proprietary model and open source model is presented in Table I.

\section{$2.4 \quad$ Pedagogical study}

Although the virtual labs platform has over 210000 registered uses, in order to assess the role of such online labs as supplementary education tools for University students and teaching material for professors, we used direct and online feedback from user communities who attended the several hands-on workshop sessions.

Evaluating students' usage perspective on remote lab as a learning tool: To analyse the role of remote robotics labs as learning material, experiments based on the 
online articulator including the control of a servo motor in a bio-robotic environment, understanding the kinematics of a robotic upper arm, were given as an exercise to a group of 60 postgraduate students (mean age $=21$ ). Participants were not previously familiar with these experiments from classroom education. Participants were trained to operate the remotely controlled experiment set-up and equipment. As feedback, a set of questions in TABLE II were provided and the student's responses were analysed. Participants rated the questions by giving Likert-scale numerical values from 1 to 5 (1Very poor, 2- Poor, 3- Average, 4- Good, 5- Excellent). The aim was to analyse whether students were able to interpret both theoretical and laboratory aspects of the experiments using the remotely controlled education platform.

Analysis of teacher perspectives: To analyse usage of remotely controlled labs in classroom education, we collected feedback from 100 university professors who attended a one-day workshop on "Blending virtual labs into the regular curriculum of educational institutes" at our campus. A comprehensive overview of usage of virtual and remote labs was provided to the participants followed by hands-on training on usage of remote equipment and robotic articulator in a blended classroom scenario. After training, a set of feedback questions (see TABLE III) regarding the remote lab usage was given to the participants. Teachers marked their choices (agree/disagree) on blending remote labs to student's curriculum.

Table 1. Cost Analysis Of Open Source And Proprietary Model Implementations Of Remote Labs

\begin{tabular}{|l|l|}
\hline Parameters chosen for cost analysis & Factors taken into account for analysis \\
\hline Experiment design and implementation & Software cost, hardware and material cost \\
\hline Deliverability of online resources & $\begin{array}{l}\text { Computers with internet connection and other network } \\
\text { devices }\end{array}$ \\
\hline Maintenance of laboratory resources & Energy cost, Component replacement, Employee cost \\
\hline
\end{tabular}

\section{RESULTS}

\subsection{Remotely controlled robotic arm as a supplementary classroom material in universities}

Among 60 students in a postgraduate classroom, 66.6\% indicated, remotely controlled experiments are an excellent tool for learning as it resembled a traditional laboratory and the experiments had a realistic effect.

$16.66 \%$ indicated it as a good material and the remaining $16.66 \%$ indicated it as an average online tool for learning. 75\% students indicated the online performance of the experiment was excellent throughout the lab hours and $70 \%$ students reported that the control of remote experiments and equipment was sufficient to understand the experimental concepts. Also, $90 \%$ of students indicated remote labs as an excellent tool for gaining a clear understanding of the experiment and related topics while $10 \%$ indicated it as a good tool for understanding concepts. Low rating in some cases correlated with lack of computer proficiency to work with remote experimentations (see Fig. 5A). 
Paper-Enabling a Freely Accessible Open Source Remotely Controlled Robotic Articulator with a Neuro-Inspired Control Algorithm

The survey involving the 100 college professors indicated that $75 \%$ preferred to include remotely controlled experiments in their classroom scenarios, $82 \%$ agreed that remote labs helps them to teach students on the basics and underlying mechanisms for practicing real labs and equipment related studies, $76 \%$ teachers indicated that control over interactions provided on experimental interface were satisfactory to supplement traditional laboratory education, while $78 \%$ indicated that the topics covered under remote laboratories were relevant to the courses that were offered at their university (Fig. 5B). Verbal feedback from the university teachers was to include more remotely controlled experiments.

Table 2. Evaluating the Effectiveness of Remotely Controlled Experiments in Classroom Education

\begin{tabular}{|c|c|}
\hline SI. No. & Questions \\
\hline Q1 & $\begin{array}{c}\text { How realistic do you feel while working with the remotely controlled experiments when } \\
\text { compared to traditional labs? }\end{array}$ \\
\hline Q2 & The online performance of the experiment was satisfactory throughout the lab hours. \\
\hline Q3 & The control of remote experiments and equipment was sufficient to understand the concepts. \\
\hline Q4 & A clear understanding of the experiment and related topics was gained \\
\hline
\end{tabular}

Table 3. Evaluating Usage Of Remote Labs In Classrooms

\begin{tabular}{|c|c|}
\hline SI. No. & Questions \\
\hline Q1 & I prefer to include remotely controlled experiments in my classroom \\
\hline Q2 & $\begin{array}{c}\text { Remote lab helps teaching students on the basics and underlying mechanisms for practicing } \\
\text { real labs and equipment based studies. }\end{array}$ \\
\hline Q3 & $\begin{array}{c}\text { The controls over the interactions on experimental interface were satisfactory to supplement } \\
\text { laboratory education }\end{array}$ \\
\hline Q4 & The topics covered were relevant to the course offered at your institute. \\
\hline
\end{tabular}
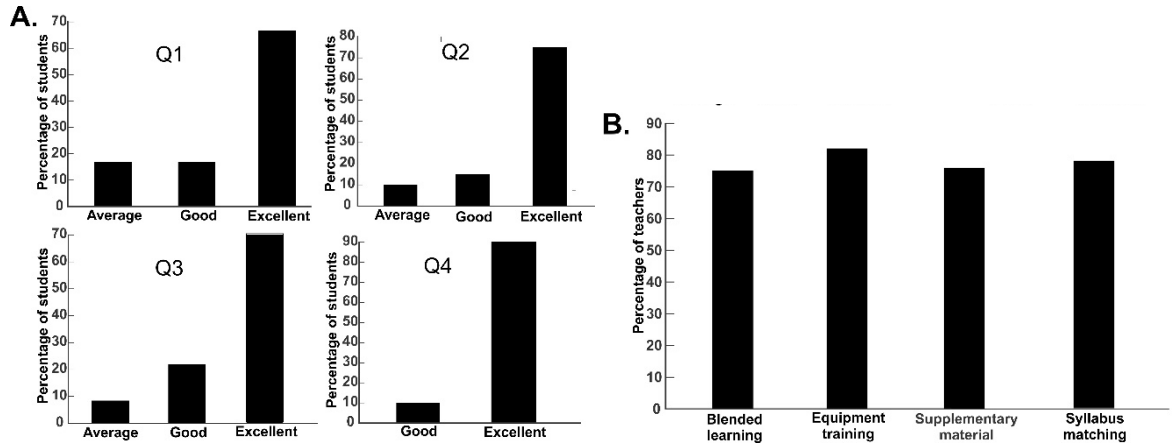

Fig. 5. Pedagogical Analysis: A. Student feedback on usage of remote laboratories in their learning. B. Teachers' responses towards usage of remote labs in their teaching curriculum. 


\subsection{Cost Benefit Analysis of Remote Lab Models}

In the FOSS architecture, a single raspberry device handled four experimental setups although commercial data acquisition device allowed better scalability for multiple experiments. For each of the experiments, device specific Java code were used. Usage of Wi-Fi adapter on Raspberry Pi made it portable.

Cost Benefit Analysis (CBA) study showed most of the experiments deployed in our laboratories were economically viable (Fig. 6) for multi-device remote labs.Costs for implementing open source model was relatively low when compared with proprietary model by avoiding licensing costs. Deliverability for ICT-employed open and commercial platform dependent methods was comparatively similar.

\subsection{Reconstruction of Kinematics from Neural Activity and Learning Behaviour}

Encoding of kinematic parameters by the cerebellum-like neural network involved firing rate dynamics (Fig. 7A). Each articulator parameter was encoded as a variation in rate code. Total simulation time for a single pattern was $1 \mathrm{~s}$. The mossy fibre input (Fig. 7A) generated using the encoding scheme was mapped to the granular layer which acts as association layer. Around 3\% of the neurons were excited (100 off 3600 neurons) for implicit representation of an input datapoint.

Employing percentage split cross-validation, accuracy was seen higher when dataset split was between 60-70\% (Fig. 7B). Mean squared error (MSE) estimates indicated that the prediction accuracy increases as the number of epochs increases and reaches an optimum solution (Fig. 7C) from an average of 0.3771 to 0.1583 .

With a Hebbian learning model and a network learning rate of 0.01 , the controller could predict and classify movement (red trajectory in Fig. 7D) and the prediction was validated using trajectory generated through $\mathrm{DH}$ transformations (blue trajectory in Fig. 7D).

Granular layer neural network model was deployed to reduce computational cost and to represent both generalization and learning interference. 4 spiking neurons were used for discretizing each input feature. Optimal results for classification were obtained when the quantization was in the range of 3-5. Average percentage accuracy was $64 \%$ and computational time $188.714 \pm 20$ s. When quantization was modified to 6 , accuracy decreased to $41 \%$ and computational time increased to $319.164 \pm 12 \mathrm{~s}$.

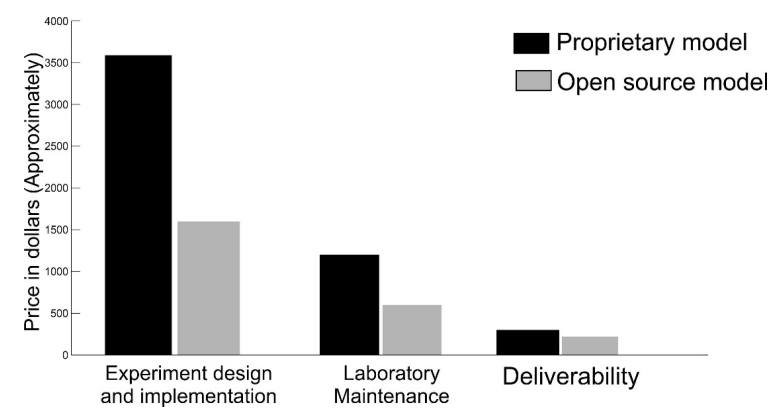

Fig. 6. Comparison of Economic cost of open source and proprietary software. 
Paper-Enabling a Freely Accessible Open Source Remotely Controlled Robotic Articulator with a Neuro-Inspired Control Algorithm

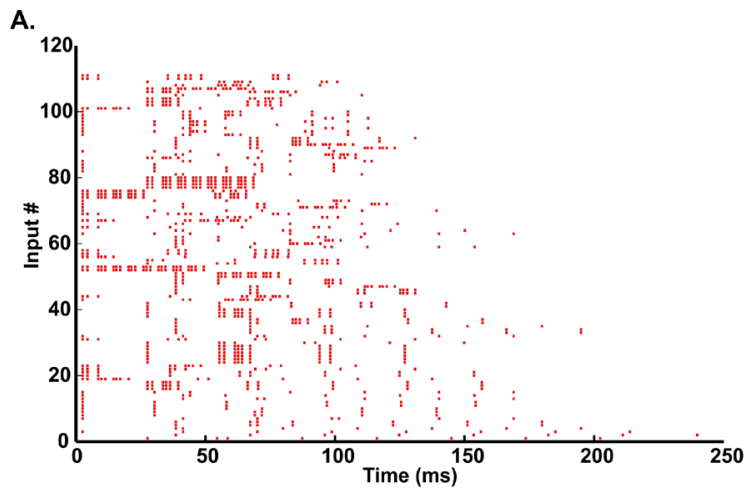

B.
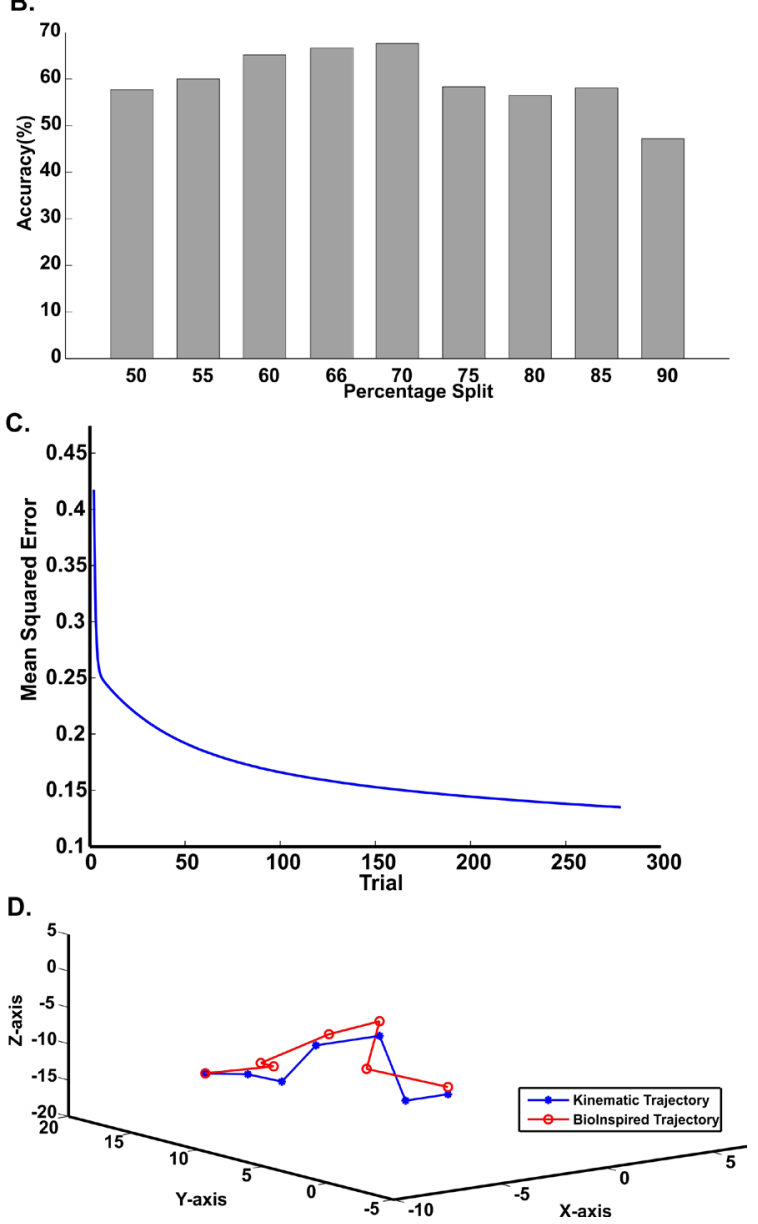

Fig. 7. Bioinspired Controller with a cerebellum-inspired architecture. A. Encoded mossy fibre behaviour from kinematic parameters. B. Percentage accuracy for dataset with different percentage split. C. Generalization behaviour of bioinspired network. D. Trajectory

$$
\text { comparison }
$$




\section{Discussion and Conclusion}

Online laboratory education with neuro-inspired controller based articulator allows a novel method to teach and learn robotics in the context of bioinspired robotics. Through our deployment we found ICT-enabled remote laboratories for robotic education are useful as learning or teaching platforms for students and teachers. Although remote labs are not favoured by biology students, we find an increasing trend in usage of such tools for skill training. Examination season showed augmented usage of the remote devices as learning environments (data not shown). Feedback collected suggested that remote labs for bioinspired robotics improved the technical performance of students taking a first course in robotics via the remote labs. Student users indicated that remote lab as a good or excellent educational tool for understanding the experiments related to robotics and its related contents. Less than $20 \%$ of the students have stated about usability, online performance and the equipment satisfaction was average. We also saw some of the correlations to poor score relating to deliverability of online resources like of the speed of the internet. $70-80 \%$ teachers reported that remote labs are used in classrooms and follow university syllabus with equipment and reference materials rated as satisfactory.

Due to difficulties like cost and infrastructure, laboratory education has become a major challenge to emerging universities. Virtual and remote laboratories are perceived by teachers as a supplement to laboratory education and skill training.

FOSS implementation based remote labs involves more equipment-specific programming but can be viewed as a cost effective method when compared to proprietary software based implementations. Our FOSS implementation could handle 4 experiments at a time but was sufficient for our purposes. At the lab, this has also encouraged student to develop remote labs during their thesis internships. We also heard more groups willing to engage in unifying several such engineering problems as online laboratories.

From the robotics perspective, this was a first demonstration of a spiking control algorithm for low-cost robotic articulators that employs a cerebellar architecture allowing neuromorphic interpretations to model sensorimotor processing in brain circuits. Synchronous firing behaviour of the input neural firing patterns suggest that the algorithm looks into the concept of generalisation and also learning interference. The role of the algorithm as a pattern separator allows the controller to be scalable. When increased DOF were to be included, number of neurons augmented linearly with the number of kinematic parameters and DOF. This will further allow more complicated robotic articulators to be controlled without major changes to the algorithm. Studies [35] demonstrate dynamical state of the network was regulate by a small number of neurons as in biological networks. In our model, around 3\% i.e. 100 of 3600 neurons are excited while representing a single data point.

From a biomimetic perspective, the model focusses on signals based on the spike timing and amount of information to kernelize the kinematics of a multi-DOF robotic articulator. The role of timing, although significant, was not included in this implementation. Adaptation of information by complex interneurons can also be incorporated to include feedforward and feedback roles to regulate movement control. We are extending this model by introducing spatio-temporal properties of spiking neurons organized 
in modular microzones and implementing learning rules at these layers to improve timing.

As a remote lab, we are yet to develop a GUI for the control algorithm and to allow users to define and manipulate the organization of the software neural network model and its learning dynamics. We are yet to build a neural interface to optimize the spiking neurons in the network. However, at the current state, we find teachers and students employing our lab for both study and dataset generation. Having remote lab on robotics has helped several groups to compete at conference competitions. Several userrelated challenges like the economic cost, anytime- anywhere access and biological resemblance are indicated to have been overcome with the deployment of remote lab implemented on a FOSS platform. We see several humanitarian technology challenges that employ robotics for education, skill training, during disaster management and for healthcare and medicine.

\section{Acknowledgment}

This project derives direction and ideas from the chancellor of Amrita University, Sri Mata Amritanandamayi Devi. This work is funded under the SR/CSRI/60/2013, SR/CSRI/61/2014 from Department of Science and Technology and the Sakshat project of National Mission on Education through Department of Higher Education, MHRD, Government of India. Authors would like to thank Rakhi Radhamani, Hareesh Singanamala, Hemalatha Sasidharakurup, Nijin Nizar for their support with this study.

\section{References}

1. S. Diwakar, K. Achuthan, and P. Nedungadi, "Biotechnology Virtual Labs - Integrating Wet-lab Techniques and Theoretical Learning for Enhanced Learning at Universities," in International Conference on Data Storage and Data Engineering, 2010, pp. 10-14. https://doi.org/10.1109/dsde.2010.22

2. K. Balasubramanian, W. Clarke-Okah, J. Daniel, F. Ferreira, A. Kanwar, A. Kwan, J. Lesperance, J. Mallet, A. Umar, and P. West, "ICTs for Higher Education," UNESCO World Conf. High. Educ., pp. 19-25, 2009.

3. J. Ma and J. V Nickerson, "Hands-on, simulated, and remote laboratories," ACM Comput. Surv., vol. 38, no. 3, p. 7-es, Sep. 2006.

4. S. Diwakar, D. Kumar, R. Radhamani, H. Sasidharakurup, N. Nizar, K. Achuthan, P. Nedungadi, R. Raman, and B. Nair, "Complementing Education via Virtual Labs: Implementation and Deployment of Remote Laboratories and Usage Analysis in South Indian Villages," Int. J. Online Eng., vol. 12, no. 3, pp. 8-15, 2016. https://doi.org/10.3991/ijoe.v12i03.5391

5. S. Diwakar, R. Radhamani, H. Sasidharakurup, D. Kumar, N. Nizar, K. Achuthan, and B. Nair, "Role of ICT-enabled Virtual Laboratories in Biotechnology Education: Case studies on blended and remote learning," in Interactive Collaborative Learning (ICL), 2015 International Conference on, 2015, no. September, pp. 915-921. https://doi.org/10.1109/icl.2015.7318149

6. H. Sasidharakurup, R. Radhamani, D. Kumar, N. Nizar, K. Achuthan, and S. Diwakar, "Using Virtual Laboratories as Interactive Textbooks: Studies on Blended Learning in Biotechnology Classrooms," EAI Endorsed Trans. e-Learning, vol. 2, no. 6, p. e4, 2015. 
7. J. Machotka and Z. Nedic, "The Remote Laboratory NetLab for Teaching Engineering Courses," Glob. J. Eng. Educ., vol. 10, no. 2, pp. 205 -212, 2006.

8. H. Kim, H. Choi, J. Han, and H. J. So, "Enhancing teachers' ICT capacity for the 21 st century learning environment: Three cases of teacher education in korea," Australas. $J$. Educ. Technol., vol. 28, no. 6, pp. 965-982, 2012. https://doi.org/10.14742/ajet.805

9. Y. Bar-Cohen and C. Breazeal, "Biologically Inspired Intelligent Robotics," in Proceedings of the SPIE Smart Structures Conference San Diego, 2003, pp. 5051-02.

10. M. Barak, "Closing the Gap Between Attitudes and Perceptions About ICT-Enhanced Learning Among Pre-service STEM Teachers," J. Sci. Educ. Technol., vol. 23, no. 1, pp. 1-14, 2014. https://doi.org/10.1007/s10956-013-9446-8

11. P. F. Santana, J. Barata, and L. Correia, "Sustainable Robots for Humanitarian Demining," Int. J. Adv. Robot. Syst., vol. 4, no. 2, pp. 1729-8806, 2007.

12. M. Kocaturk, H. O. Gulcur, and R. Canbeyli, "Toward building hybrid biological/in silico neural networks for motor neuroprosthetic control," Front. Neurorobot., vol. 9, no. AUG, pp. 1-21, 2015. https://doi.org/10.3389/fnbot.2015.00008

13. M. C. Mozer, "Neural network speech processing for toys and consumer electronics," IEEE Expert, vol. 11, pp. 4-5, 1996.

14. A. Frosini, M. Gori, and P. Priami, "A neural network-based model for paper currency recognition and verification," IEEE Trans. Neural Networks, vol. 7, no. 6, pp. 1482-1490, 1996. https://doi.org/10.1109/72.548175

15. J. S. Albus, "A New Approach to Manipulator Control: The Cerebellar Model Articulation Controller(CMAC)," J. Dyn. Syst. Meas. Control, 1975. https://doi.org/10.1115/1.3426922

16. M. Kawato, "Internal models for motor control and trajectory planning," Curr. Opin. Neurobiol., vol. 9, pp. 718-727, 1999. https://doi.org/10.1016/S0959-4388(99)00028-8

17. A. Zbrzeski, Y. Bornat, B. Hillen, R. Siu, J. Abbas, R. Jung, and S. Renaud, "Bio-inspired Controller on an FPGA applied to Closed-loop Diaphragmatic Stimulation," Front. Neurosci., vol. 10, no. June, p. 275, 2016. https://doi.org/10.3389/fnins.2016.00275

18. R. A. Miranda, W. D. Casebeer, A. M. Hein, J. W. Judy, E. P. Krotkov, T. L. Laabs, J. E. Manzo, K. G. Pankartz, G. A. Pratt, J. C. Sanchez, D. J. Weber, T. L. Wheeler, and G. S. F. Ling, "DARPA-funded efforts in the development of novel brain-computer interface technologies," J. Neurosci. Methods, vol. 244, no. 2015, pp. 52-67, 2014.

19. M. Mano, G. Capi, N. Tanaka, and S. Kawahara, "An Artificial Neural Network Based Robot Controller that Uses Rat's Brain Signals," Robotics, vol. 2, no. 2, pp. 54-65, 2013. https://doi.org/10.3390/robotics2020054

20. H. Gomi and M. Kawato, "Neural network control for a closed-loop System using Feedback-error-learning," Neural Networks, vol. 6, no. 7, pp. 933-946, Jan. 1993. https://doi.org/10.1016/S0893-6080(09)80004-X

21. R. Hogri, S. A. Bamford, A. H. Taub, A. Magal, P. Del Giudice, and M. Mintz, "A neuroinspired model-based closed-loop neuroprosthesis for the substitution of a cerebellar learning function in anesthetized rats.," Sci. Rep., vol. 5, p. 8451, 2015. https://doi.org/10.1038/srep08451

22. J. DENAVIT, "A kinematic notation for lower-pair mechanisms based on matrices.," Trans. ASME. J. Appl. Mech., vol. 22, pp. 215-221, 1955.

23. S. Kucuk and Z. Bingul, "Robot Kinematics: Forward and Inverse Kinematics," in Industrial-Robotics-Theory-Modelling-Control, 2006, no. December, pp. 117-148.

24. A. K. Mitra, "Joint Motion-based Homogeneous Matrix Method for Forward Kinematic Analysis of Serial Mechanisms," Int. J. Emerg. Technol. Adv. Eng., vol. 2, no. 5, pp. 111$122,2012$. 
25. J. Iqbal, R. Islam, and H. Khan, "Modeling and Analysis of a 6 DOF Robotic Arm Manipulator," Canadian Journal on Electrical and Electronics Engineering, vol. 3, no. 6. 2012.

26. S. Sahu, B. B. Biswal, and B. Subudhi, "Computational Intelligence, Control and Automation A Novel Method for Representing Robot Kinematics using Quaternion Theory A Novel Method for Representing Robot Kinematics using Quaternion Theory," IEEE Sponsored Conference on Computational Intelligence, Control and Computer Vision in Robotics and Automation. pp. 76-82, 2008.

27. P. Nedungadi, Ra. Raman, K. Achuthan, and S. Diwakar, "Virtual Labs Collaborative \& Accessibility Platform (VLCAP)," Proc. 2011 IAJC-ASEE Int. Conf., 2011.

28. J. Freeman, A. Nagarajan, M. Parangan, D. Kumar, S. Diwakar, and K. Achuthan, "Remote triggered photovoltaic solar cell lab: Effective implementation strategies for Virtual Labs," in Technology Enhanced Education (ICTEE), 2012 IEEE International Conference on, 2012, pp. 1-7. https://doi.org/10.1109/ictee.2012.6208653

29. D. Kumar, H. Singanamala, K. Achuthan, S. Srivastava, B. Nair, and S. Diwakar, "Implementing a Remote-Triggered Light Microscope: Enabling Lab Access via Virtual labs," in Interdisciplinary Advances in Applied Computing - ICONIAAC '14,Proceedings of the 2014 International Conference on, 2014, pp. 1-6. https://doi.org/10.1145/ 2660859.2660963

30. E. D'Angelo, T. Nieus, A. Maffei, S. Armano, P. Rossi, V. Taglietti, A. Fontana, G. Naldi, and E. D. Angelo, "Theta-frequency bursting and resonance in cerebellar granule cells: experimental evidence and modeling of a slow k+-dependent mechanism.," $J$ Neurosci, vol. 21 , no. 3 , pp. $759-770,2001$.

31. R. Naud, N. Marcille, C. Clopath, and W. Gerstner, "Firing patterns in the adaptive exponential integrate-and-fire model.," Biol. Cybern., vol. 99, no. 4-5, pp. 335-347, Nov. 2008. https://doi.org/10.1007/s00422-008-0264-7

32. C. Medini, A. Vijayan, R. M. Zacharia, L. P. Rajagopal, B. Nair, and S. Diwakar, "Spike Encoding for Pattern Recognition: Comparing Cerebellum Granular Layer Encoding and BSA algorithms," in Advances in Computing, Communications and Informatics (ICACCI), 2015 International Conference on, 2015, pp. 1619-1625. https://doi.org/10.1109/icacci. 2015.7275845

33. C. Medini, S. Subramaniyam, B. Nair, and S. Diwakar, "Modeling cerebellar granular layer excitability and combinatorial computation with spikes," in Bio-Inspired Computing: Theories and Applications (BIC-TA), 2010 IEEE Fifth International Conference on, 2010, pp. 1495-1503. https://doi.org/10.1109/bicta.2010.5645274

34. K. Achuthan and S. S. Murali, "A Comparative Study of Educational Laboratories from Cost \& Learning Effectiveness Perspective," in Software Engineering in Intelligent Systems, 2015, pp. 143-153.

35. C. Clopath, J. P. Nadal, and N. Brunel, "Storage of correlated patterns in standard and bistable Purkinje cell models," PLoS Comput. Biol., vol. 8, no. 4, pp. 1-10, 2012. https://doi.org/10.1371/journal.pcbi.1002448

\section{AUTHORS}

Asha Vijayan is currently working as Assistant Professor at Amrita University (Amrita School of Biotechnology, Amrita Vishwa Vidyapeetham, Amritapuri, Clappana P.O., Kollam, 690 525, Kerala, India).

Chaitanya Nutakki is currently a Junior Research Fellow working on bio-inspired robotics at the Computational Neuroscience Lab, Amrita School of Biotechnology, 

Neuro-Inspired Control Algorithm

Amrita Vishwa Vidyapeetham (Amrita University), Amritapuri, Clappana P.O., Kollam, 690 525, Kerala, India.

Dhanush Kumar is currently working as Development Engineer in VALUE Virtual labs and is based at the Computational Neuroscience Lab of Amrita University (Amrita School of Biotechnology, Amrita Vishwa Vidyapeetham (Amrita University), Amritapuri, Clappana P.O., Kollam, 690 525, Kerala, India).

Krishnashree Achuthan is the Professor and Dean, Postgraduate Programs at Amrita University. She is also the Principal Institute Coordinator for Virtual Labs project funded by Ministry of HRD's National Mission on Education through ICT at Amrita University. She is also the Directior of Technology Business Incubator and the Center for Cyber-security, which has been recognized as a TIFAC center of excellence by Government of India (e-mail: krishna@amrita.edu).

Bipin Nair is the Professor and Dean of School of Biotechnology, Amrita Vishwa Vidyapeetham University, India. He is also the Discipline-wise National Coordinator for the development of Biotechnology and biomedical engineering Virtual Labs being developed under the MHRD -NMEICT program. (e-mail: bipin@amrita.edu).

Shyam Diwakar (corresponding author) is Associate Professor and Lab Director of Computational Neuroscience Laboratory at the School of Biotechnology, Amrita University, India. He is the Institute Intergration Coordinator for Amrita University in the National mission project on Virtual Laboratories and a Co-investigator of VALUE (Virtual and Accessible Laboratories for Universalizing Education); a virtual labs initiative supported by Sakshat mission of MHRD, Government of India, and Principle Investigator of few other projects funded by Department of Science and Technology and Department of Biotechnology, Government of India. (e-mail: shyam@amrita.edu).

This work is funded under the Sakshat project of National Mission on Education through ICT (Phase I and Phase II), Department of Higher Education, MHRD, Government of India. It is an extended and modified version of a paper presented at the the Interactive Mobile and Computer Aided Learning (IMCL2016), held in November 2016, in San Diego, CA, USA. Submitted, 22 September 2016. Published as resubmitted by the authors on 24 October 2016 